[5] E. Goursat, $A$ course in mathematical analysis, translated by E. R. Hedrick and O. Dunkel, Ginn and Co., Boston, 1917, vol. 2

[6] M. Baker and J. L. Ericksen, Inequalities restricting the form of the stress-deformation relations for isotropic elastic solids and Reiner-Rivlin fluids, J. Wash. Acad. Sci. 44, 33-35 (1954)

[7] J. G. Oldroyd, Rectilinear plastic flow of a Bingham solid. III. A more general discussion of steady flow, Proc. Cambr. Phil. Soc. 44, 200-213 (1948)

[8] J. G. Oldroyd, Rectilinear flow of non-Bingham plastic solids and non-Newtonian viscous liquids. I, Proc. Cambr. Phil. Soc. 45, 595-611 (1949)

[9] J. G. Oldroyd, Rectilinear flow of non-Bingham plastic solids and non-Newtonian viscons liquids. II, Proc. Cambr. Phil. Soc. 47, 575-584 (1951)

\title{
VECTOR FIELDS ASSOCIATED WITH PLANE PLASTICITY*
}

By D. R. BLAND (King's College, London)

Synopsis. A symmetric tensor can be associated with a vector field by means of Eq. (1). It is found that, for the stress field in plane plasticity, associated vector fields can always be found. For certain vector fields, associated stress fields exist. Examples are given.

Transformation between stress and vector. The most general way in two dimensions that the symmetric stress tensor $\sigma_{i i}$ can be associated with a vector field $v_{i}$, when no derivatives or integrals of $v_{i}$ are included, is

$$
\sigma_{i j}=M \delta_{i j}+N v_{i} v_{i}
$$

where $M$ and $N$ are functions of $v_{k}^{2}$. The stress tensor satisfies $\sigma_{i i, j}=0$. Hence, from (1),

$$
2 M^{\prime} v_{k} v_{k, i}+2 N^{\prime} v_{k} v_{k, j} v_{i} v_{i}+N v_{i, j} v_{i}+N v_{i} v_{i, i}=0 .
$$

Resolving the vector equation (2) parallel and normal to $v_{i}$,

$$
\left(2 M^{\prime}+2 N^{\prime} v_{k}^{2}+N\right) v_{i} v_{i} v_{i, i}+N v_{i}^{2} v_{i, j}=0
$$

and **

$$
\epsilon_{i l} v_{i} v_{l}\left(2 M^{\prime} v_{i, i}+N v_{i, i}\right)=0 .
$$

The invariants of the stress tensor can be found in terms of $M, N$ and $v_{k}^{2}$,

$$
I_{1}=\sigma_{i i}=2 M+N v_{k}^{2}
$$

and

$$
I_{2}=\sigma_{i j} \sigma_{i j}=2 M^{2}+2 M N v_{k}^{2}+N^{2}\left(v_{k}^{2}\right)^{2} .
$$

The stress tensor for a solid in the plastic state satisfies a criterion of the form

$$
f\left(I_{1}, I_{2}\right)=0 \text {. }
$$

Substituting from (5) and (6),

$$
g\left(M, N, v_{k}^{2}\right)=0,
$$

${ }^{*}$ Received Sept. 28, 1955; revised manuscript received November 18, 1955.

${ }^{* *} \epsilon_{i j}$ is the tensor $\epsilon_{11}=\epsilon_{22}=0, \epsilon_{12}=1, \epsilon_{21}=-1$. 
where $g$ is a known function of $M, N$ and $v_{k}^{2}$. It follows that, with the stress field for a solid in the plastic state, an infinity of vector fields satisfying the Eqs. (3) and (4) can be associated. $M$ and $N$ are functions of $v_{k}^{2}$ and satisfy Eq. (8); one of $M$ and $N$ can always be chosen arbitrarily.

With a vector field, a stress field can only be associated if the governing equations of the vector field can be expressed in the form of Eqs. (3) and (4). $M$ and $N$ are completely determined except for an arbitrary multiplicative constant and an arbitrary additive constant in $M$. This determines the associated stress field and yield criterion to within two arbitrary constants.

Two types of vector field are of particular interest, the non-dilatational and the irrotational. For the former $v_{i, i}=0$; from (3)

$$
2 M^{\prime}+2 N^{\prime} v_{k}^{2}+N=0 .
$$

The other governing equation for $v_{i}$ is, from (4),

$$
\epsilon_{i l} v_{i} v_{l}\left\{N v_{i, i}-\left(N+2 N^{\prime} v_{k}^{2}\right) v_{i, i}\right\}=0 .
$$

For the latter $v_{i, i}=v_{i, i} ;$ from (4),

$$
2 M^{\prime}+N=0
$$

and the other governing differential equation is, from (3),

$$
2 N^{\prime} v_{i} v_{j} v_{i, j}+N v_{i, j}=0 .
$$

Examples of associated fields. (i) The irrotational non-dilatational vector field $v_{i, j}=$ $v_{i, i}$ and $v_{i, i}=0$. By (9) and (11), $N=B$ and $M=-\frac{1}{2} B v_{k}^{2}+A$ where $A$ and $B$ are constants. The associated stress field is $\sigma_{i j}=\left(-\frac{1}{2} B v_{k}^{2}+A\right) \delta_{i j}+B v_{i} v_{i}$. The invariant $I_{1}=2 A$. This vector field has an associated stress field whose yield criterion is $I_{1}=$ constant.

(ii) Compressible homenergic homentropic fluid flow. The governing differential equations are $\left(\rho v_{i}\right),{ }_{i}=0, p,_{i}+\rho v_{i} v_{i, j}=0, \frac{1}{2} v_{i}^{2}+\int(1 / \rho) d p=h$ and $p=p(\rho) . v_{i}$ is the fluid velocity, $p$ the pressure and $\rho$ the density. $h$ is constant. From these equations $v_{i, j}=v_{i, i}$ and $v_{i} v_{j} v_{i, j}-(d p / d \rho) v_{i, i}=0$. On comparison with (12), it is seen that the associated stress field exists. $N\left(v_{k}^{2}\right)$ is found by eliminating $p$ and $\rho$ from $d p / d \rho=-$ $N / 2 N^{\prime}, v_{k}^{2}=2\left(h-\int(1 / \rho) d p\right)$ and $p=p(\rho)$. The transformation can be expressed in the form $\sigma_{i j}=C\left(p-p_{0}\right) \delta_{i j}+C \rho v_{i} v_{i}$, where $C$ and $p_{0}$ are constants. This transformation has been considered by Inoue [1] and Hill [2].

(iii) Shallow water flow at constant head. The governing differential equations [3] are $v_{j} v_{i, j}=-g z_{, i},\left(z v_{i}\right)_{, i}=0$ and $g\left(z_{0}-z\right)=\frac{1}{2} v_{i}^{2} . v_{i}$ is velocity and $z$ depth. $z_{0}$ is constant. Whence $v_{i, j}=v_{i, i}$ and $v_{i} v_{i} v_{i, j}-\left(g z_{0}-\frac{1}{2} v_{k}^{2}\right) v_{i, i}=0$. On comparison with (12) it is seen that the associated stress field exists. The transformation is $\sigma_{i i}=\left(A+\frac{1}{2} B g^{2} z^{2}\right)$ $\delta_{i j}+B g z v_{i} v_{i}$, where $A$ and $B$ are constants. The associated yield criterion is

$$
\left(I_{1}-\sqrt{\frac{I_{2}^{\prime}}{2}}-2 A\right)^{2}=B g^{2} z_{0}^{2}\left(I_{1}-2 \sqrt{\frac{I_{2}^{\prime}}{2}}-2 A\right) .
$$

(iv) Plane strain of a Mises or Tresca solid. The yield criterion is $2 I_{2}-I_{1}^{2}=K^{2}$, constant. From (5) and (6), $N=k / v_{k}^{2}$. The associated non-dilatational vector field will 
be $v_{i, i}=0$ and $\epsilon_{i l} v_{j} v_{l}\left(v_{i, j}+v_{i, i}=0\right.$ with the transformation

$$
\sigma_{i i}=\left(\frac{K}{2} \ln v_{k}^{2}+C\right) \delta_{i i}+\frac{K}{v_{k}^{2}} v_{i} v_{i} .
$$

(v) Plane stress of a Mises solid. The yield criterion is $3 I_{2}-I_{1}^{2}=2 K^{2}$. Substitution from (5) and (6) shows that $M$ and $N$ satisfy

$$
M^{2}+M N v_{k}^{2}+N^{2}\left(v_{k}^{2}\right)^{2}=K^{2} .
$$

\section{REFERENCES}

1. N. Inoue, 1952, J. Phys. Soc. Japan, 7, 119 and 518 (1952); J. Aero. Sci., 19, 783 (1952); Doshisha Eng. Review, Special Paper No. 1 (1952).

2. R. Hill, J. Mech. Phys. Solids 2, 110 (1954).

3. Ernst Preiswerk, Nat. Advisory Comm. Aeronaut. Tech. Mem. No. 934, (1940).

\section{PHYSICAL INTERPRETATION OF PHYSICAL COMPONENTS OF STRESS AND STRAIN*}

\section{BY DANIEL FREDERICK (Virginia Polytechnic Institute)}

1. Introduction. The purpose of this paper is to give a physical interpretation to the several different sets of physical components of stress and strain which have been introduced in the literature by different authors and to present and interpret some new sets. The question of the physical interpretation of the physical components of stress and strain arises when one expresses the equations of mechanics involving these in a general coordinate system through the use of tensor calculus. Since the tensor form of the equations can be obtained readily, it is of practical interest to have a direct physical interpretation of the physical components which are related to the tensor quantities.

The reader is assumed to be familiar with the elements of tensor calculus as presented in the book by Synge and Schild [1] and their basic notation will be used here. All quantities are referred to the ordinary physical space which is flat and has a positive definite metric form.

2. The physical components of the stress tensor. These will be introduced through the equations of equilibrium using the four sets of components of the stress vectors which act on the faces of an infinitesimal curvilinear tetrahedron associated with the covariant (contravariant) base vectors $\bar{g}_{i}\left(\bar{g}^{i}\right)$. When these equilibrium equations are compared with corresponding tensor equations, four different sets of physical components arise for each triad.

(a) Physical components of a vector. The four sets of physical components associated with a vector $\bar{Z}$ in general coordinates $x^{r}$ will be divided into two groups. The first are called orthogonal components, since they are obtained by projecting the vector orthogonally onto the base vectors, and will be denoted by the capital letter $O$ to the left of the base letter. Those components along the covariant or contravariant base triads which add by the vector or parallelogram law to give the original vector are called parallelogram components. They are marked with the capital letter $P$ to the left of the base letter. Used as subscripts (superscripts) these letters refer to components along

\footnotetext{
*Received July 15, 1955; revised manuscript received October 5, 1955.
} 